\title{
Integrated Observation of Multi-Channel Ground-Based Lidar and Space- Based Lidar in Nanjing
}

\section{Cao $N^{1 *}$, Xie $Y^{1 *}$, Wang $X^{1}$, Song $X Y^{1}$ and Zhu $C^{1}$}

${ }^{1}$ Key Laboratory of Aerosol-Cloud Precipitation of China Meteorological Administration, Nanjing University of Information Science and Technology Nanjing, Jiangsu 210044, China

${ }^{2}$ Qinghai Institute of Meteorological Science, Xining, Qinghai 810000, China

\begin{abstract}
This paper introduces the structure and working principle of Mie-Rayleigh-Raman lidar, observes and compares the Nanjing aerosol of Mie channel with that of the Raman channel respectively. It shows that there is some uncertainty about lidar boundary parameters, however, Raman scattering lidar could overcome such problems effectively that we often use multi-channel Mie-Rayleigh-Raman lidar. The observation results obtained by groundbased Mie-Rayleigh-Raman lidar are consistent with that from space-based lidar, and the former is more accurate than the latter. Integrated observation of the two will be the main direction of the development of aerosol observation in the future.
\end{abstract}

Keywords: Lidar; Aerosol; Scattering

\section{Introduction}

Atmospheric aerosol is closely related to human activities as a main component of atmosphere and its main sources are volcanic eruptions and other natural disasters, industrial emissions, car exhaust, dust pollution and so on. It has been a hot topic that increasing aerosols result in global warming and climate change, fine particles such as PM2.5 and PM10 aerosols trigger a variety of respiratory diseases at the same time which have been a serious threat to human health in recent years. To control and reduce emissions of aerosols, it is all based on the aerosol observations whatever the means adopted. There are three categories to observe aerosol so far, satellite, aerial and ground observation, and ground observation includes sun photometer and radar which is good at observing smoke, fog, clouds, temperature, dust storms and haze [1]. And it is widely used as its simple operation, high resolution and accuracy. Traditional lidar has simple structure and mature technology, but there are some uncertainty in determining the boundary value, something about the wavelength, and extinction backscatter ratio. Raman lidar has an advantage of reducing parameter assumption which not only has higher precision but also can solve the above problems [2]. Its echo signal is so weak and signal to noise ratio is not easy to control that we usually use statistics or dichotomy to overcome such problems; We usually use photon counting and cooling methods to reduce Raman lidar observation error and noise restrictions. We usually use the Mie channel and Raman channel of Mie-Rayleigh-Raman lidar to measure aerosol. Mie channel is also widely used to measure the cloud, and Rayleigh channel is used to observe temperature. Because of the satellite is used as a platform, space-based lidar has wide observation field of view and high orbit with high sensitivity and high resolution. In this paper, we use a multichannel Mie-Rayleigh-Raman lidar to observe aerosols in Nanjing, inverse aerosol in Mie channel and Raman channel respectively, and compare the observation results with that from space-based lidar.

\section{Mie-Rayleigh-Raman Lidar System}

Mie-Rayleigh-Raman lidar is multi-channel lidar system as is shown in Figure 1. Laser transmitter emission wavelength is $532 \mathrm{~nm}$, the laser emit upward into the atmosphere through a beam expander, occurs in the atmosphere and its backscatter echo signal is received by telescope. Raman scattering signal in nitrogen molecules $(607 \mathrm{~nm})$ enters into Raman photon counting acquisition channel through the dichroic mirror. Since Raman signal is weak, in order to prevent damage to low-altitude $532 \mathrm{~nm}$ signal detector, we have to use a gate signal to control Raman signal acquisition channel; $532 \mathrm{nmH}$ scattering signal through a half mirror enters into Rayleigh photon counting acquisition channel, and high-altitude signal often delays than that at low altitude, so the channel is also controlled by a gate signal which receives the low-altitude Mie scattering signal; The processed scattering signal can be converted into an electrical signal through a photovoltaic system, and is stored in the host computer by the data acquisition system ultimately.

The signal echo strength in Mie channel, Raman channel and Rayleigh channel has a time delay as is shown in Figure 2. Time delay is designed to protect the photomultiplier tube is not damaged by high-intensity echo. In general, low-altitude Mie scattering echo is very strong, but the experimental system use A/D converter in Mie channel, so the detector cannot easily be destroyed. Rayleigh channel and Raman channel use photomultiplier tube to measure faint echo energy, so time delay can effectively ensure the weak signal detection. High-intensity signal at low altitude will not be accepted in order to protect the safety of the use of two-channel detector.

\section{Experimental Observations}

\section{Observations of haze}

The moon imaging shoot at 20:00 on $10^{\text {th }}$ December, 2011 in the northern suburb of Nanjing is shown in Figure 3a. It's sunny weather,

*Corresponding author: Nianwen Cao, key laboratory of Aerosol-Cloud Precipitation of China Meteorological Administration, Nanjing University of Information Science and Technology Nanjing, Jiangsu 210044, China, Tel: +862558731101; E-mail: nwcao@nuist.edu.cn

Received November 11, 2016; Accepted December 27, 2016; Published December 30, 2016

Citation: Cao N, Xie Y, Wang X, Song XY, Zhu C (2016) Integrated Observation of Multi-Channel Ground-Based Lidar and Space-Based Lidar in Nanjing. J Laser Opt Photonics 3: 145. doi: 10.4172/2469-410X.1000145

Copyright: (C) 2016 Cao N, et al. This is an open-access article distributed under the terms of the Creative Commons Attribution License, which permits unrestricted use, distribution, and reproduction in any medium, provided the original author and source are credited. 
Citation: Cao N, Xie Y, Wang X, Song XY, Zhu C (2016) Integrated Observation of Multi-Channel Ground-Based Lidar and Space-Based Lidar in Nanjing. J Laser Opt Photonics 3: 145. doi: 10.4172/2469-410X.1000145

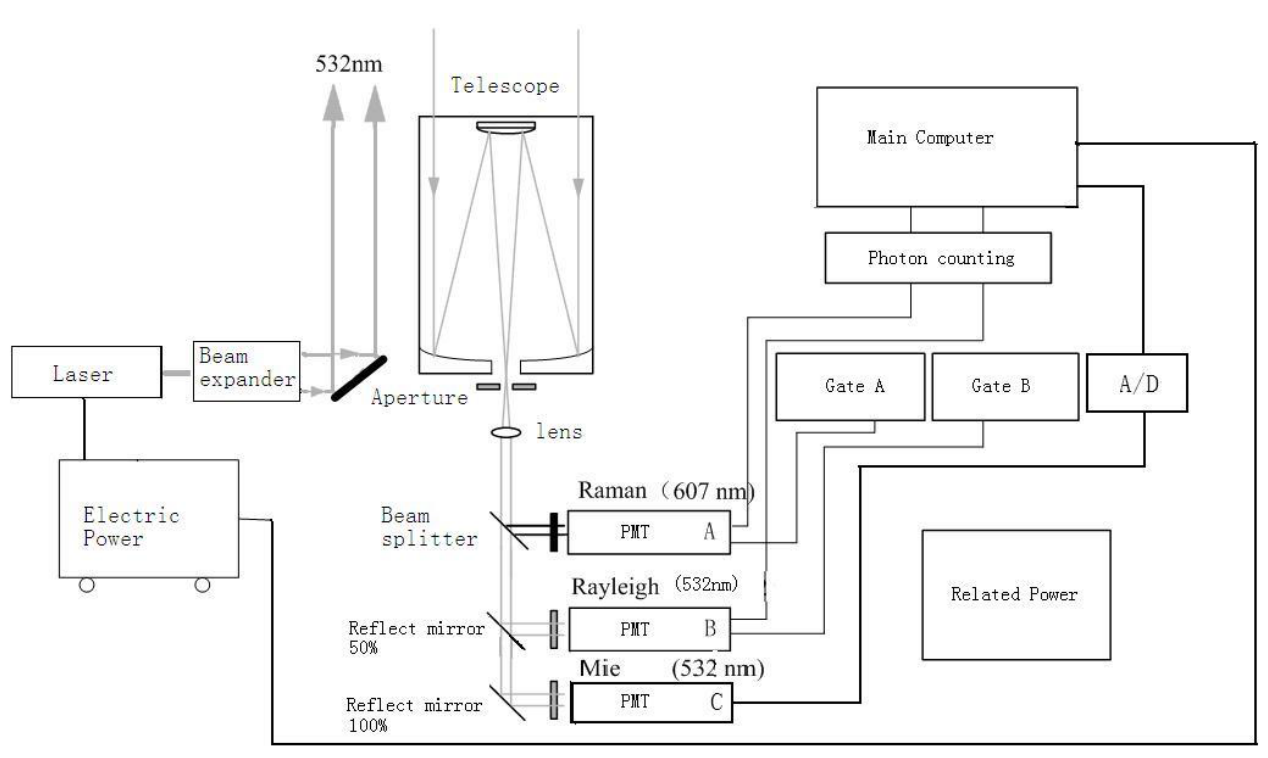

Figure 1: The structure of Mie-Rayleigh-Raman lidar system.
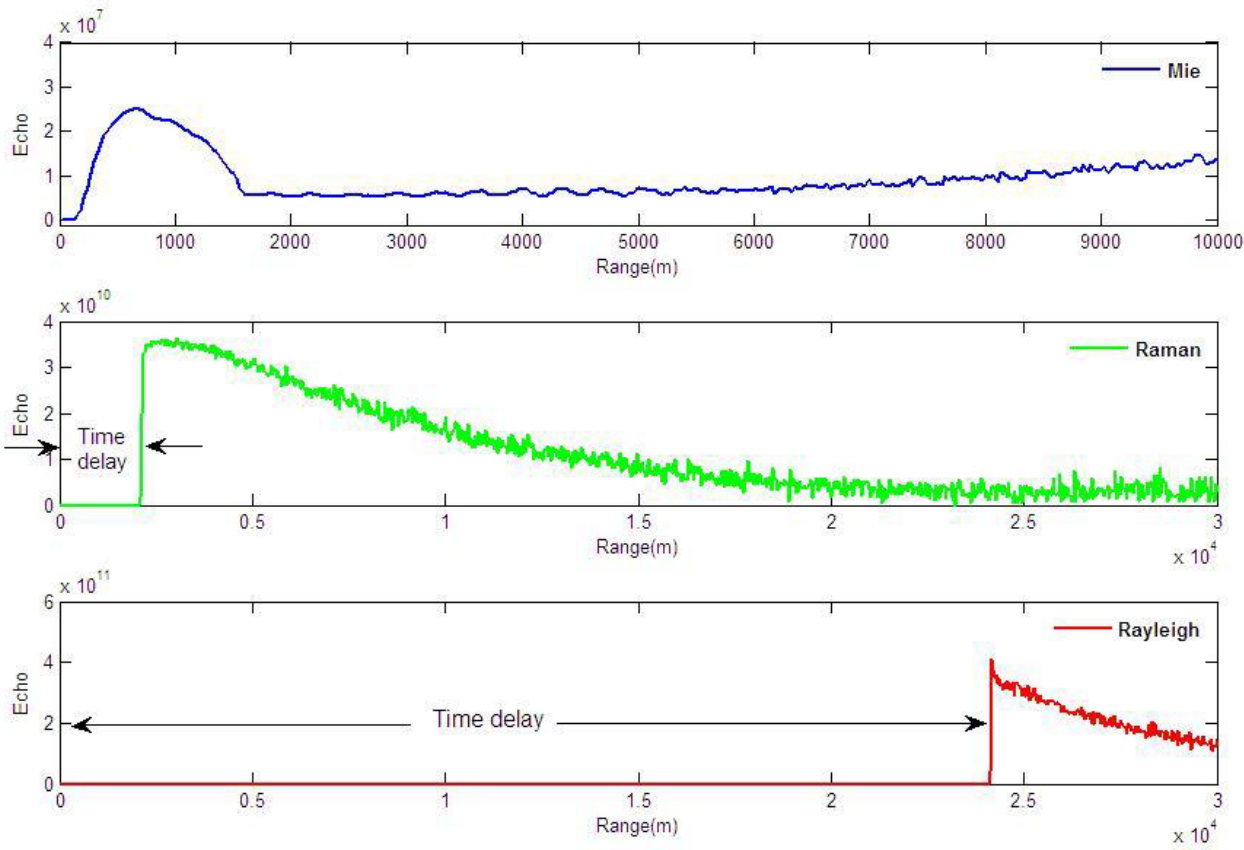

Figure 2: The echo strength of Mie-Rayleigh-Raman channel lidar system.

good air quality and clear image of the moon indicates scattering is small. As is shown in Figure $3 \mathrm{~b}$, there is fog around the moon and the moon imaging shoot is blurred at 17:00 on $4^{\text {th }}$ April, 2012, indicating that the forward scattering and scattering around is strong, backscattering is small. The greater the concentration of the fog, the stronger the forward scattering and the scattering around the smaller the backscattering.

As is shown in Figure 4, the continuous radar observations of haze at 1:00 to 19:00 pm on $24^{\text {th }}$ October, 2009 indicate haze is light. However, it has suffered more serious layered haze from about 10 o'clock at 300 meters below. The weather forecasts said that it was a sunny day at 8:00, there was fog at 9-10:00 and there was haze at 1019:00. It shows that lidar observations of haze are consistent with that said in weather forecast and lidar is effective and precise in inversing aerosols.

\section{High precision inversion of Mie scattering of the aerosols}

We present five different boundary values when using lidar to inverse and calculate extinction coefficient a, $1.4 \times 10^{-5}, 1.2 \times 10^{-5}, 1 \times$ $10^{-5}, 0.8 \times 10^{-5}, 0.6 \times 10^{-5}$, as is shown in Figure 5. As is shown in Figure 
Citation: Cao N, Xie Y, Wang X, Song XY, Zhu C (2016) Integrated Observation of Multi-Channel Ground-Based Lidar and Space-Based Lidar in Nanjing. J Laser Opt Photonics 3: 145. doi: 10.4172/2469-410X.1000145

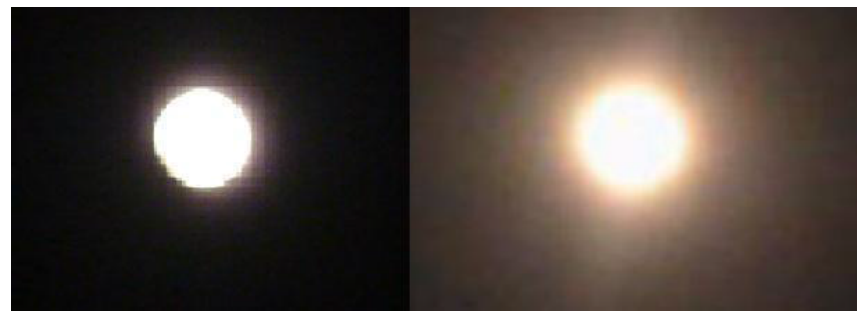

Figure 3: The moon imaging shoot, a: at 20:00 on 10th December, 2011, sunny, b: at 17:00 on 4th February 2011, foggy.

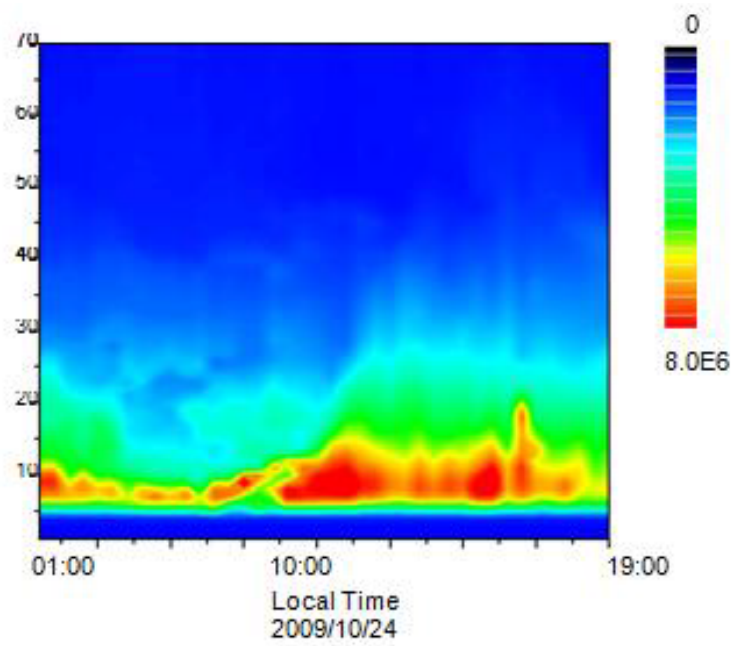

Figure 4: The continuous radar observations of haze on October 24, 2009

$5 \mathrm{a}$, the profiles over $2 \mathrm{~km}$ are almost overlapping, but there is great difference between the calculated inversion value below $2 \mathrm{~km}$; it can be found that there are a few bumps in Figure 5b, and the gap between the extinction coefficient is large in place of these projections. Therefore, we can conclude that, the inversion results will be less affected by the boundary value when occurs at high altitude; but the inversion results are very sensitive to the choice of the reference point because of the elements of the low atmosphere is unstable which is subject to weather conditions. We select the observation data of 20 October 2011 evening as an example and inverse by Fernald with Mie channel echo signal to obtain a set of aerosol extinction coefficient profiles as is shown in Figure $6 \mathrm{a}-6 \mathrm{~d}$. There are 19 groups of inversion results of Mie-RayleighRaman lidar observation data in picture above. There are $5 \times 3$ groups of 10000 pulses observation data inversion results in Figure 6a-6c. There are $4 \times 1$ group of 10000 pulses observation data inversion results in Figure 6d. The inversion results of Mie-Rayleigh-Raman lidar (RRML) show that the extinction coefficient is high from 19:00 to 19:17 at about $4 \sim 5.5 \mathrm{~km}$., It can be regarded as the clouds when at the height of mixed boundary layer or above; At 18:51 20:04, the extinction coefficient is about $0.1 \mathrm{~km}^{-1}$ within the range of the boundary layer height, but it is not large at the height of $2 \sim 4 \mathrm{~km}$; After 20:37, the extinction coefficient decreases with increasing height within $2 \mathrm{~km}$, but the overall value is smaller than that of haze days; From 19:43, we can observe extinction coefficient profiles increase to peak at $4 \mathrm{~km} \sim 5 \mathrm{~km}$, and the peak becomes more apparent over time from 20:37. Extinction coefficient peak becomes even more obvious even bimodal or multimodal, and the impact of the range of height is $3 \mathrm{~km} \sim 7 \mathrm{~km}$. Based on the query to display, there is haze at 18:00 19:00 and it is cloudy after 20:00,
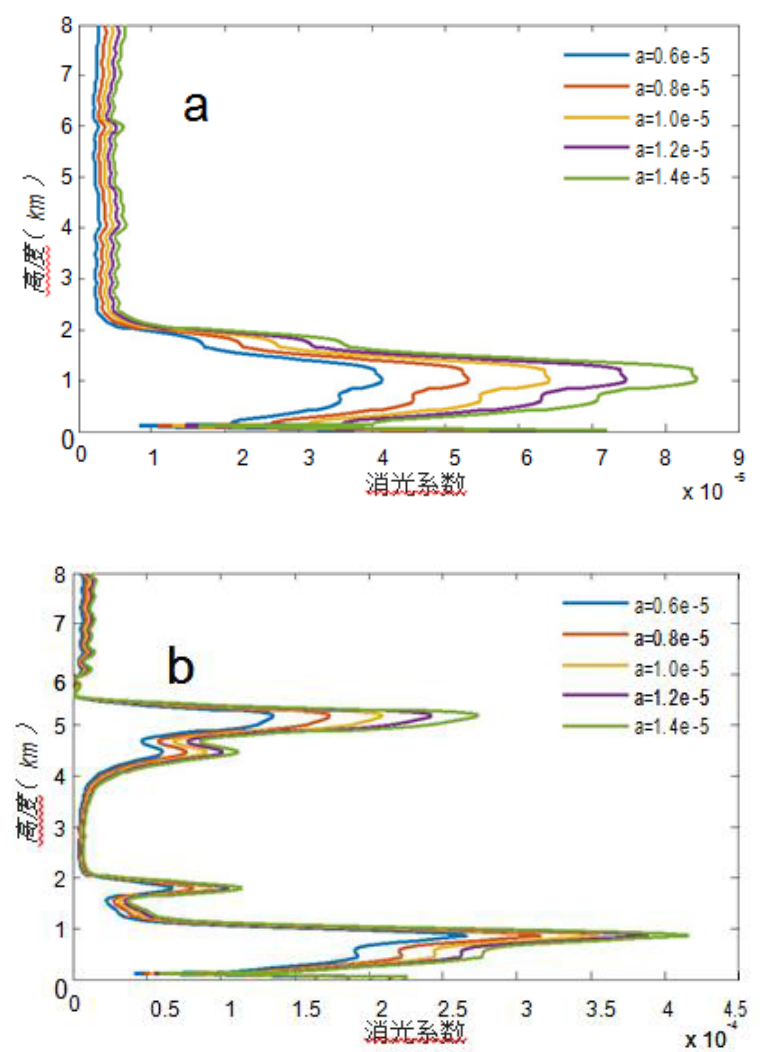

Figure 5: Extinction coefficient profiles of different boundary value inversion.

which is consistent with the experimental observations. Therefore MieRayleigh-Raman lidar observations can found clouds and information about height and thickness of cloud by extinction coefficient, and because of its time resolution is higher than ground observation, we can observe something ignored when ground observation, such as the clouds move faster and so on.

\section{Comparative analysis of Mie scattering lidar and Raman scattering lidar observation}

We use data from Mie channel and Raman channel at the same time to compare and analyze the inversion results of Raman scattering and Mie scattering. It shows that the receiving signal field of two channels is consistent. Then we inverse the extinction coefficient and backscattering coefficient respectively, as is shown in Figures 7 and 8.

As is shown in Figure 7, the trend of Mie scattering signal is consistent with that of Raman scattering signal, and the inversion profiles is smoother after wavelet de-noising processing. As is shown in Figure $7 \mathrm{~b}$, both the peak of profiles appears at about $2.7 \mathrm{~km}$. The maximum of Raman scattering extinction coefficient could be up to $0.17 \mathrm{~km}^{-1}$, and Mie scattering extinction coefficient could be up to $0.25 \mathrm{~km}^{-1}$. Due to its height higher than the boundary layer, it can be considered as aerosol groups or clouds. This is consistent with backscattering coefficient profile in Figure $8 \mathrm{~b}$, and the peak height is also about $2.7 \mathrm{~km}$. As is shown in Figure 7c, maximum of Raman scattering extinction coefficient could be up to $0.5 \mathrm{~km}^{-1}$, maximum of Mie scattering extinction coefficient could be up to $1.15 \mathrm{~km}^{-1}$, both profiles of the peak have emerged at about $3.5 \mathrm{~km}$ and $4.5 \mathrm{~km}$ due to 

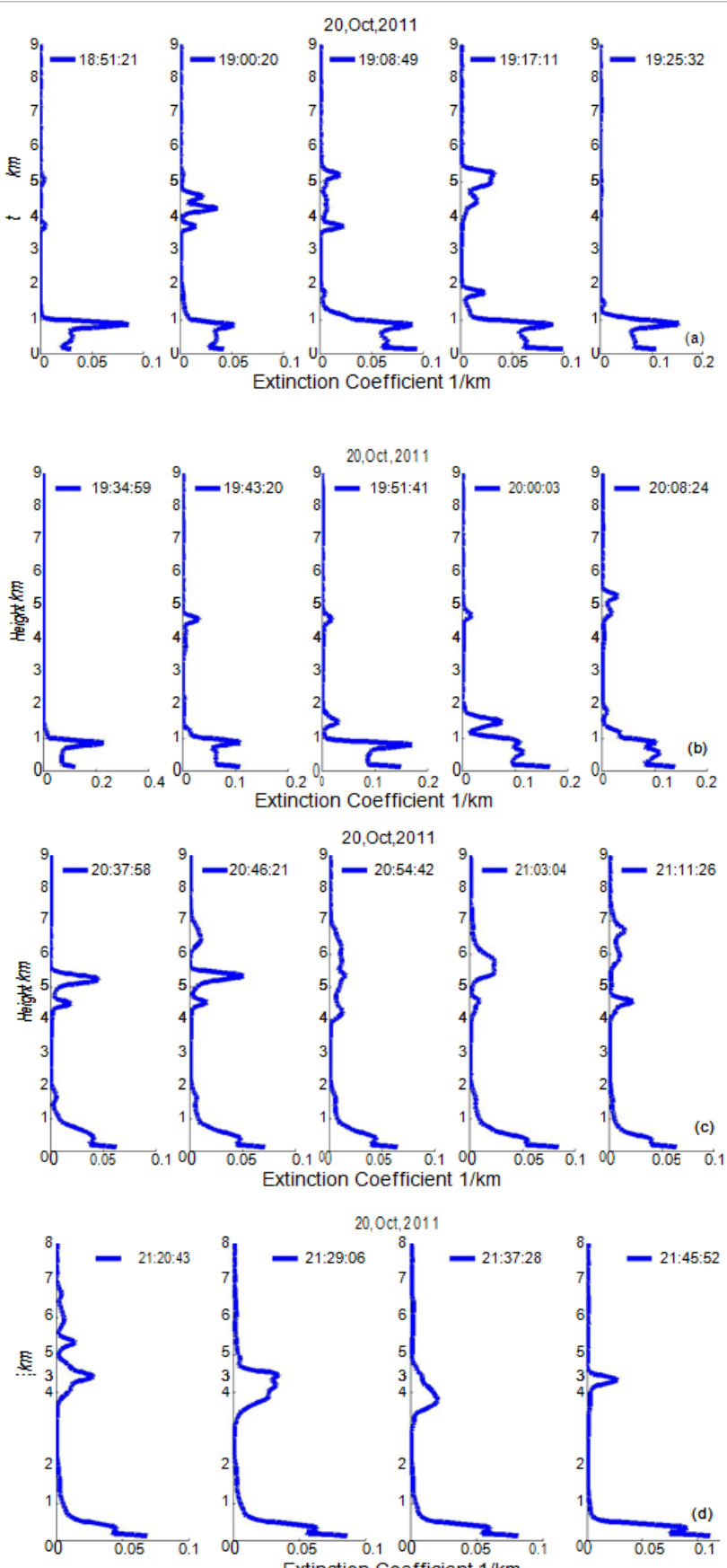

Figure 6: RRML actual vertical profiles of aerosol extinction coefficient.

the coefficient becomes large. Since the extinction coefficient decreases rapidly above mixing boundary layer height, it can be seen that layer of clouds is thick, attenuation of the laser is strong and the laser could not penetrate clouds. The two peaks of backscattering coefficient profiles also appear at $3.5 \mathrm{~km}$ and $4.5 \mathrm{~km}$ which means Mie-Rayleigh-Raman lidar system can observe clouds.

\section{Comparison of Detection of Spaced-Based Lidar and Ground-Based Lidar}

Cloud aerosol lidar and infrared pathfinder satellite observation (CALIPSO) is mounted on CALIOP which can real-time observe the
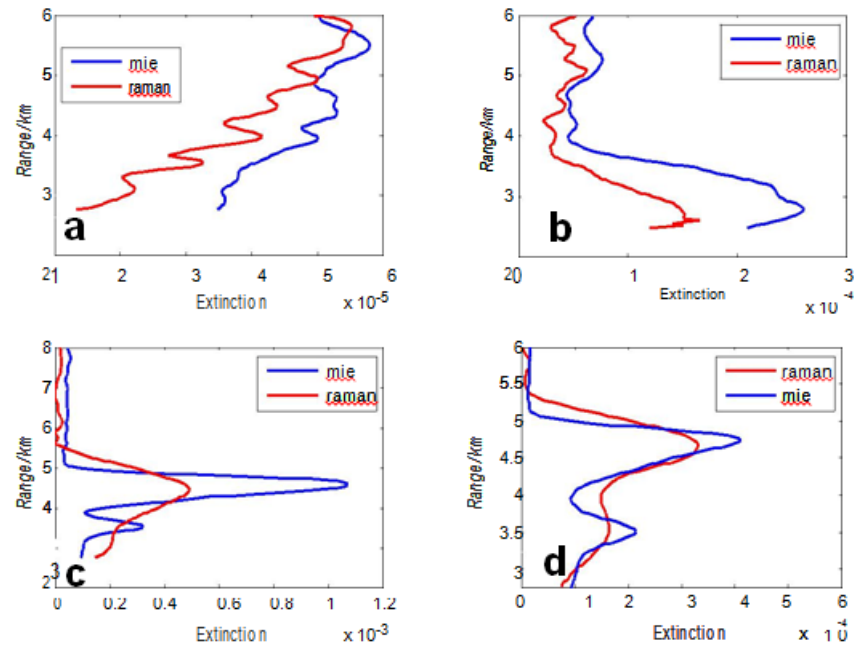

Figure 7: Comparison of inversion of aerosol extinction coefficient of Raman signal and Mie signal.
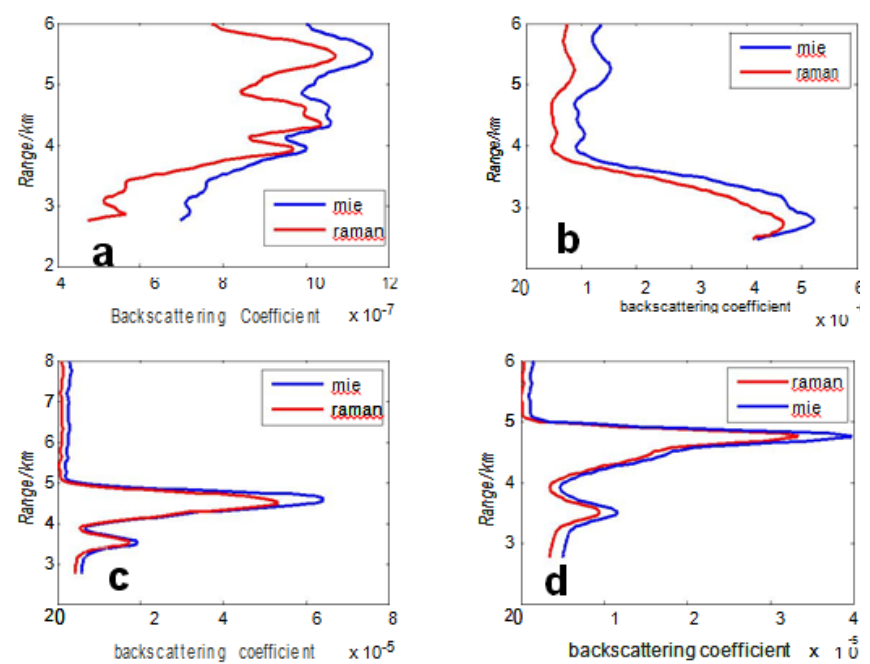

Figure 8: Comparison of inversion of aerosol backscattering coefficient of Raman signal and Mie signal.

global atmospheric aerosols fast and continuously. Due to satellite orbit of CALIPSO is narrow, it will not match the ground-based lidar exactly when transiting. We select a study area to obtain aerosol optical properties and physical parameters using CALIPSO transiting many times to inverse data within a certain time. What is shown in Figure $9 \mathrm{a}$ and $9 \mathrm{~b}$ is the track of Yangtze River CALIPSO transiting nadir in November, 2013 (14 groups) and December, 2013 (15 groups whose range is $30^{\circ} \mathrm{N} \sim 35^{\circ} \mathrm{N}$ and $116^{\circ} \mathrm{E} \sim 120^{\circ} \mathrm{E}$. The red trace is track of nadir when transiting in the daytime, blue trace is track of nadir when transiting at night; the green point is the matched area for the selection and placement of Mie-Rayleigh-Raman laser in the experimental observations. Due to the time resolution of the data increases, we select diamond region cross trace of day and night (Figure 9) .

Figures 10 and 11 shows the vertical profiles of average extinction coefficient in Nanjing and the surrounding area when there is haze in November 2013 and December 2013. 


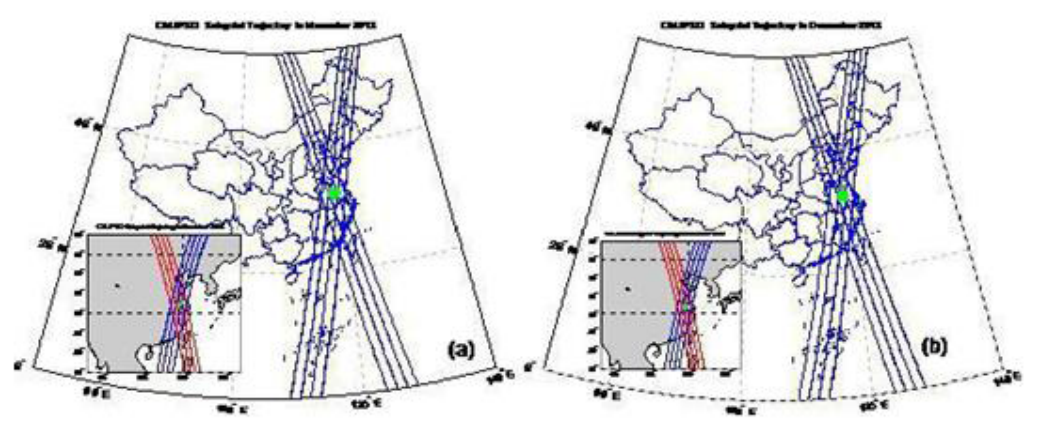

Figure 9: Yangtze River Delta-satellite ground track and ground-based radar station location on November and December in 2013.

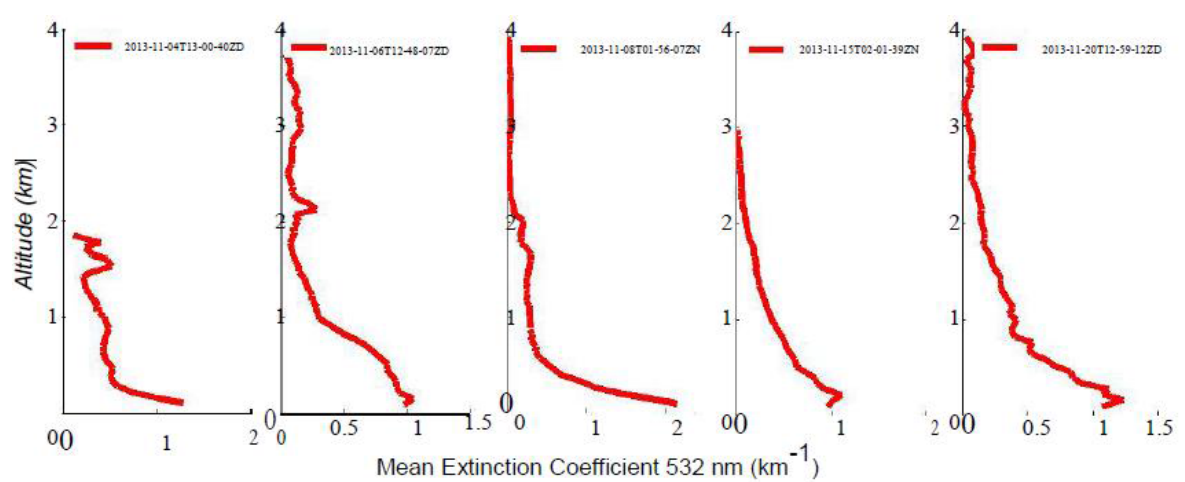

Figure 10: Average extinction coefficient near Ground area $532 \mathrm{~nm}$ when there is haze in November 2013.

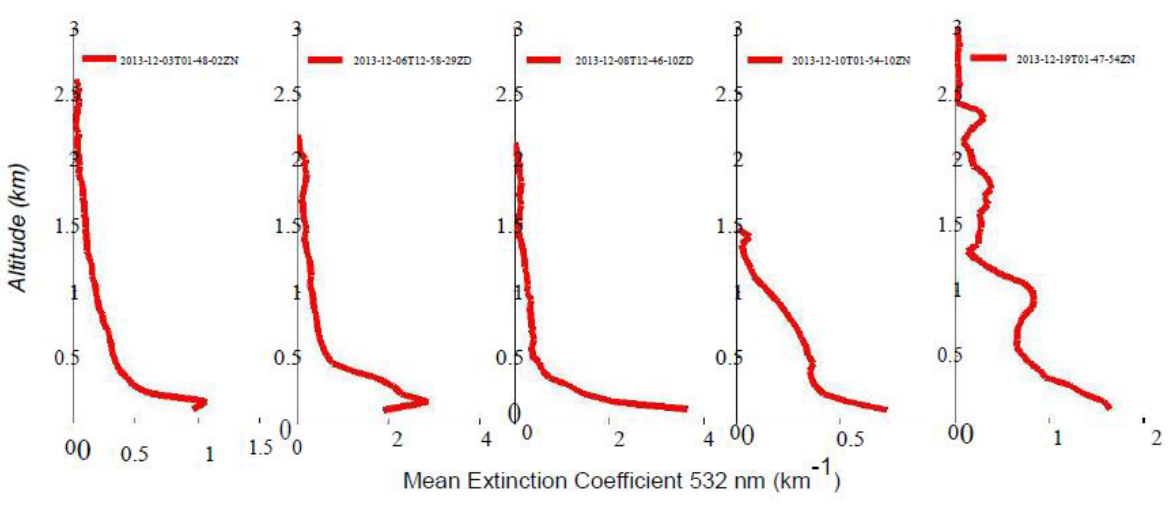

Figure 11: Average extinction coefficient near Ground area $532 \mathrm{~nm}$ when there is haze in December 2013.

As is shown in Figures 10 and 11 the average aerosol extinction coefficient decreases with increasing altitude when there is haze. And the value coincides with the results from lidar, the maximum value of the extinction coefficient could be up to $3.8 \mathrm{~km}^{-1}$. CALIPSO observation to earth is continuous scanning. Although the track is narrow, it still be able to get the extinction coefficient of the vertical height on the inner track. Figure 12a-12e are the vertical distribution of extinction coefficient in the CALIPSO satellite orbit where ground-based lidar is located.

Figure 12a-12e are the vertical distribution of aerosol extinction coefficient in $30^{\circ} \mathrm{N} 35^{\circ} \mathrm{N}$ Satellite orbit. There is a small value empty window area of extinction coefficient in the vertical height between $30.5 \sim 31.5^{\circ} \mathrm{N}$ which is the range of Anhui Dabie Mountain area, from latitude $30^{\circ} 10^{\prime}$ to $32^{\circ} 30^{\prime}$, from longitude $112^{\circ} 40^{\prime}$ to $117^{\circ} 10^{\prime}$. The average altitude of the mountain is about $1000 \mathrm{~m}$, the maximum is 1770 $\mathrm{m}$, the maximum of steepness of the mountain is $50^{\circ}$. Due to Dabie Mountain area is of high forest cover, pollution-free and good air quality, CALIPSO vertical distribution value of extinction coefficient in the region is small. The article also analyzes the once dust weather process from 27 to 29 in November, 2012 in Nanjing for selecting the appropriate satellite transiting time and the satellite nadir track to get a track closer to Nanjing to analyze in the period of dust when satellite transiting.

Since CALIPSO operation cycle is 16 days, we select a nadir track closer to Nanjing in the world map as is shown in Figure 14a and intercept local map of the Yangtze River Delta. (b). The red line 
Citation: Cao N, Xie Y, Wang X, Song XY, Zhu C (2016) Integrated Observation of Multi-Channel Ground-Based Lidar and Space-Based Lidar in Nanjing. J Laser Opt Photonics 3: 145. doi: 10.4172/2469-410X.1000145
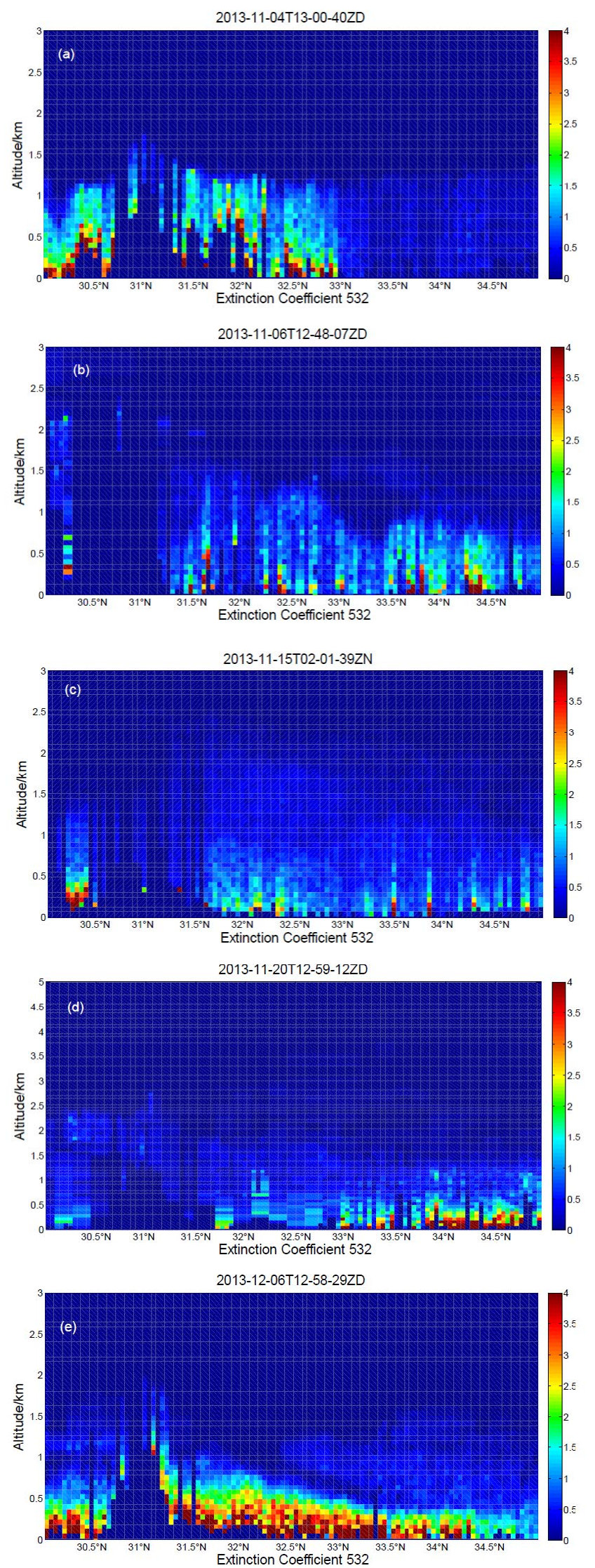

Figure 12(a-e): The vertical distribution of aerosol extinction coefficient in $30^{\circ} \mathrm{N} \sim 35^{\circ} \mathrm{N}$ Satellite orbit. 
Citation: Cao N, Xie Y, Wang X, Song XY, Zhu C (2016) Integrated Observation of Multi-Channel Ground-Based Lidar and Space-Based Lidar in Nanjing. J Laser Opt Photonics 3: 145. doi: 10.4172/2469-410X.1000145

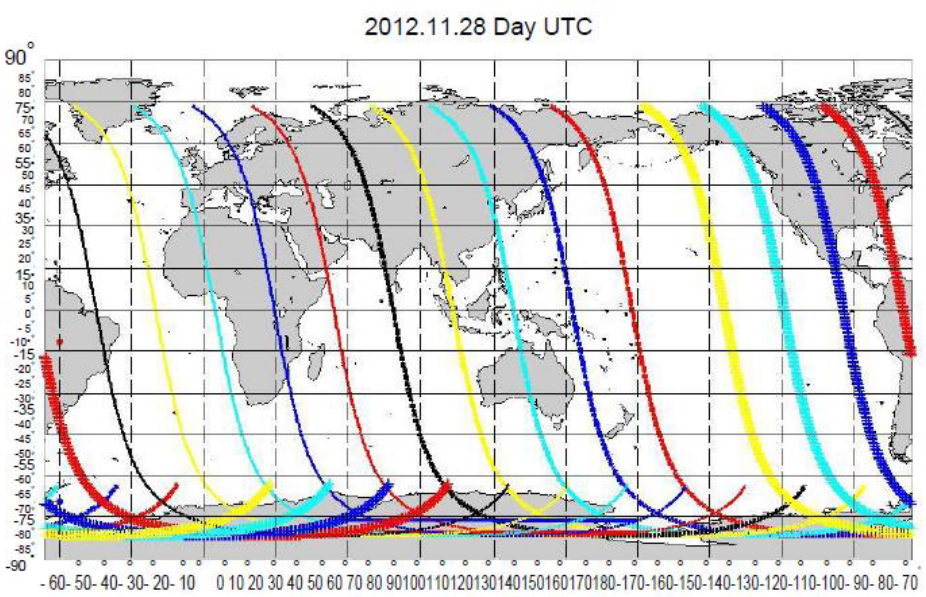

Figure 13: CALIPSO Groundtrack.
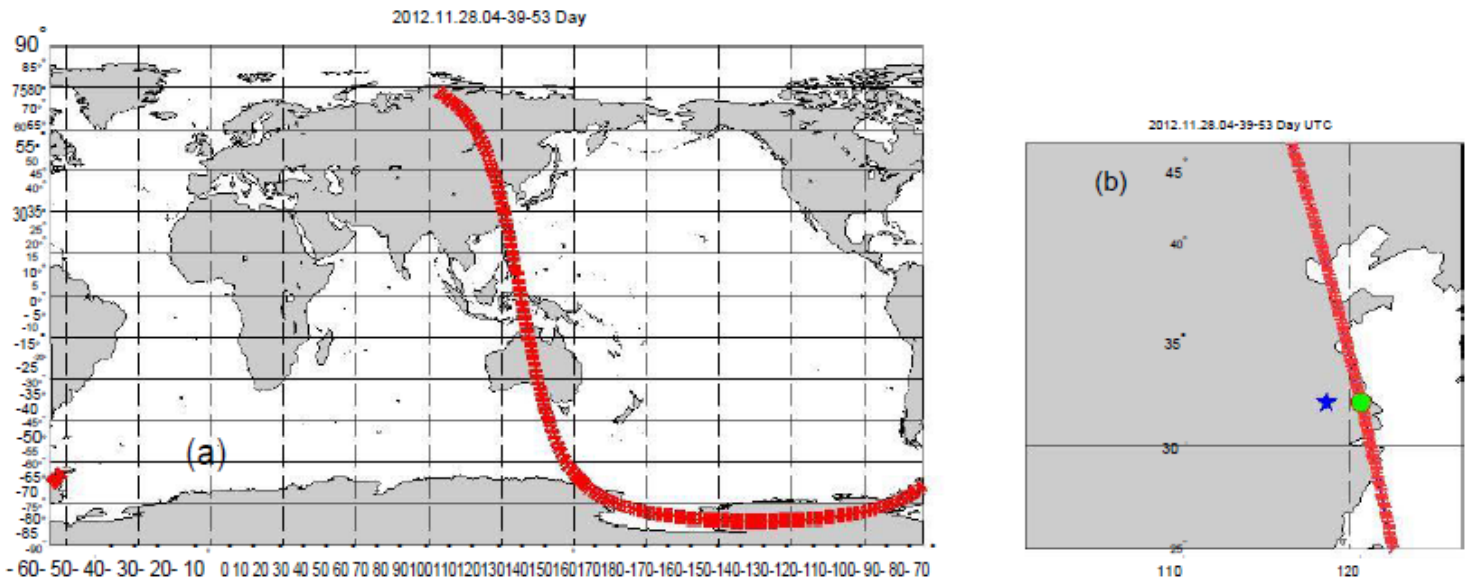

Figure 14: Satellite ground track and RRML station location when transiting Nanjing on $28^{\text {th }}$ November, 2012.

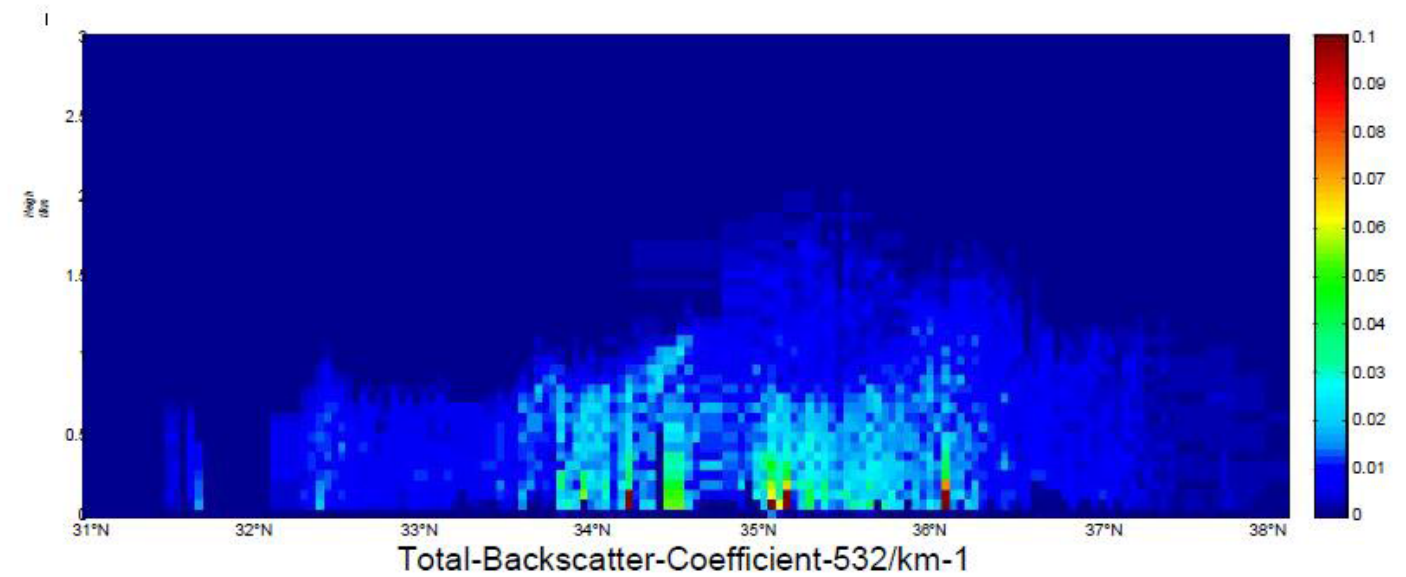

Figure 15: The vertical distribution of backscatter coefficient at $31^{\circ} \mathrm{N} \sim 38{ }^{\circ} \mathrm{N}$ track tape.

in Figure $14 \mathrm{~b}$ is the satellite nadir track, five-pointed star represents ground-based laser radar observation stations, and dots indicate the satellite nadir nearest to ground-based laser radar.
Figures 15 and 16 are the vertical distribution of the aerosol backscatter coefficient and extinction coefficient of $31^{\circ} \mathrm{N} \sim 38^{\circ} \mathrm{N}$ track tape when there is dust, the maximum of extinction coefficient could 
Citation: Cao N, Xie Y, Wang X, Song XY, Zhu C (2016) Integrated Observation of Multi-Channel Ground-Based Lidar and Space-Based Lidar in Nanjing. J Laser Opt Photonics 3: 145. doi: 10.4172/2469-410X.1000145

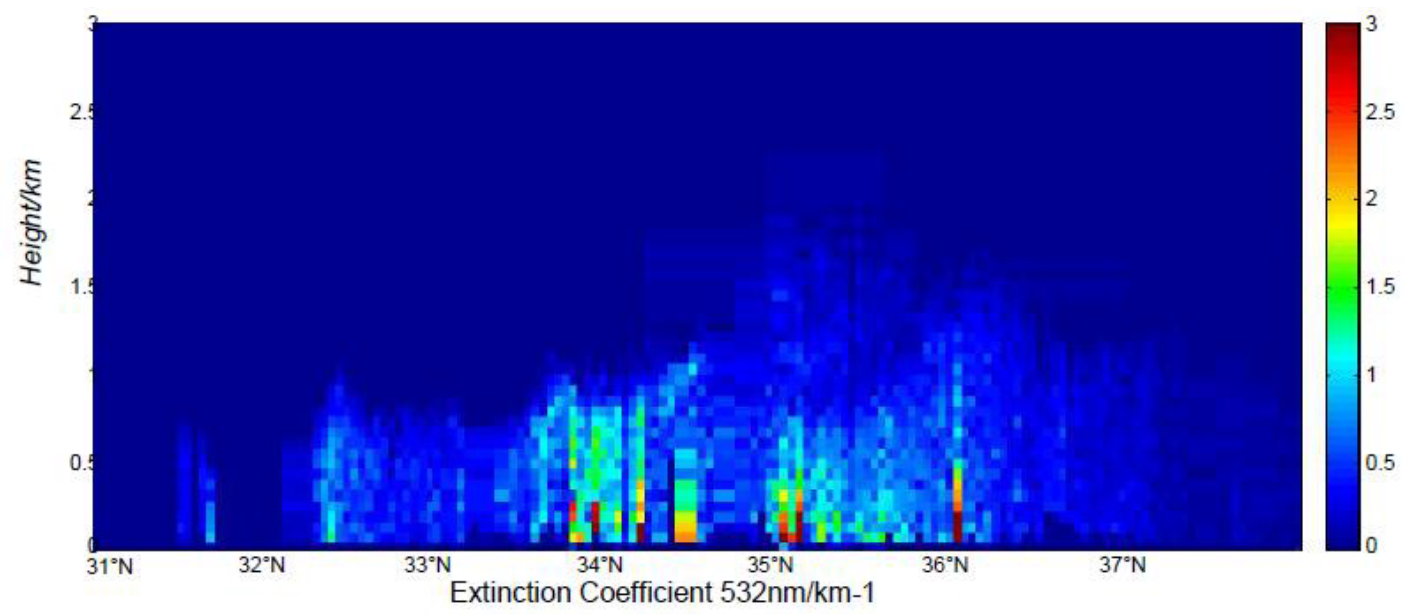

Figure 16: Vertical distribution of extinction coefficient on $31^{\circ} \mathrm{N} \sim 38^{\circ} \mathrm{NTrack}$ tape.

be up to $3 \mathrm{~km}^{-1}$, the average height of the dust impact is about $1 \mathrm{~km}$ and the maximum of it is $1.5 \mathrm{~km}$, which is consistent with the real-time monitoring results of micro-pulse polarization lidar in the northern suburb of Nanjing. It makes up for the limitations of single-station ground-based laser radar observation. We can monitor the dynamics of dust storms more effectively if combining ground-based lidar with space-based lidar.

\section{Conclusion}

In this paper, we observe Nanjing aerosol with multi-channel MieRayleigh-Raman and inverse signal in Mie channel and Raman channel respectively. It shows that: Mie scattering lidar have some uncertainty in determining the boundary value, correlation to wavelength and extinction to backscatter ratio, but Raman lidar have no need to determine boundary value. So multi-channel Mie-Rayleigh-Raman lidar is a powerful tool to observe aerosol. The aerosol observation results by space-based lidar are consistent with that by multi-channel ground-based lidar. Integrated observations of multi-channel groundbased lidar and space-based lidar can effectively compensate the lack of the both two so as to observe aerosol more efficiently and accurately.

\section{Acknowledgements}

This work was supported by National Science Foundation of China under Project 41375044/D0503 and 41175033/D0503.

\section{References}

1. Gang L, Weizhe S, Rui Y (2008) Cloud-Aerosol Lidar of America. Spacecraft Engineering 17: 78-84.

2. Cunxiong Z, Nianwen C, Fengkai $Y$ (2015) Micro Pulse Lidar Observations of Aerosols in Nanjing. Laser and Optoelec Progr 5: 16-22. 